\title{
Cantilever-based bio-chemical sensor integrated in a microliquid handling system
}

Thaysen, Jacob; Marie, Rodolphe; Boisen, Anja

Published in:

Proceedings of The 14th IEEE International Conference on Micro Electro Mechanical Systems

Link to article, DOI:

10.1109/MEMSYS.2001.906561

Publication date:

2001

Document Version

Publisher's PDF, also known as Version of record

Link back to DTU Orbit

Citation (APA):

Thaysen, J., Marie, R., \& Boisen, A. (2001). Cantilever-based bio-chemical sensor integrated in a microliquid handling system. In Proceedings of The 14th IEEE International Conference on Micro Electro Mechanical Systems (pp. 401-404). IEEE. https://doi.org/10.1109/MEMSYS.2001.906561

\section{General rights}

Copyright and moral rights for the publications made accessible in the public portal are retained by the authors and/or other copyright owners and it is a condition of accessing publications that users recognise and abide by the legal requirements associated with these rights.

- Users may download and print one copy of any publication from the public portal for the purpose of private study or research.

- You may not further distribute the material or use it for any profit-making activity or commercial gain

- You may freely distribute the URL identifying the publication in the public portal 


\title{
CANTILEVER-BASED BIO-CHEMICAL SENSOR INTEGRATED IN A MICROLIQUID HANDLING SYSTEM
}

\author{
J. Thaysen, R. Marie, and A. Boisen \\ Mikroelektronik Centret, Bldg. 345e, Technical University of Denmark \\ DK-2800 Kgs. Lyngby, Denmark
}

\begin{abstract}
ABSTRAC'T
We present a new cantilever-based bio-chemical sensor integrated in a microliquid handling system. The cantilevers have integrated piezoresistive readout which, compared to optical readout, enables simple measurements on even non-transparent liquids, such as blood. First, we introduce a simple theory for using piezoresistive cantilevers as surface stress sensors. Then, the sensor fabrication based on conventional microfabrication is described and the sensor characterization is discussed. During the characterization we found a stress sensitivity of $(\Delta R / R)=4.6 \cdot 10^{-4}(\mathrm{~N} / \mathrm{m})^{-1}$ and a minimum detectable surface stress change of $2.6 \mathrm{mN} / \mathrm{m}$. Aqua regia etch of gold on top of the cantilevers has been monitored, and immobilization of single-stranded thiol modified DNA-oligos has been detected by the sensor. Finally, it is demonstrated that it is possible to analyze two samples simultaneously by utilizing the laminar flow in the microliquid handling system.
\end{abstract}

\section{INTRODUCTION}

Several groups have investigated cantilever-based biochemcial sensors in the recent years $[1,2,3]$. J. Fritz et al. [1] have for example demonstrated sensitive detection of DNA hybridization where DNA sequences, which differ by only a single base, can be distinguished. The cantilever-based sensor is very small, sensitive and portable and it is thus possible to use the sensor in, for example point-of-care in-vitro diagnostics.

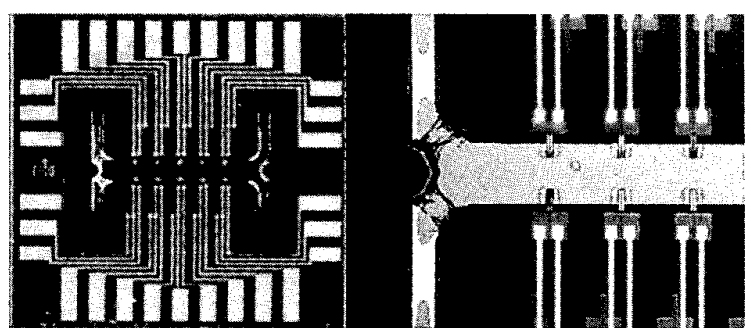

Figure 1: Optical microscope images of the sensor chip. The left picture shows the whole chip, which consists of 10 cantilevers in a channel and 4 inlets/outlets. The right picture shows a part of the channel, where six cantilevers are visible.

In cantilever-based sensing, cantilevers designed for atomic force microscopy are normally used. The cantilevers are placed in liquid cells with volumes of hundreds of microliters. When molecules bind to the cantilever surface, the cantilever will start to bend. The cantilever bending is traditionally monitored by optical techniques. Using optical detection, it is difficult to perform simultaneous measurements on several cantilevers since each cantilever in principle needs its own optical readout system. Moreover, measurements can only be performed on transparent liquid samples. Finally, the large volume liquid cells allow temperature gradients and turbulences to exits in the liquid. This will result in drift and noise in the sensor signal.

Our sensor consists of 10 cantilevers with piezoresistive readout placed in a small channel having a volume of only $0.14 \mu 1$, see figure 1. No turbulence is possible in the liquid channel and temperature gradients are minimized significantly. Moreover, the chip is designed with four inlets/outlets. This allows us to flow two liquid samples through the channel at the same time.

The total chip size is $6 \times 6 \mathrm{~mm}^{2}$ and the piezoresistive cantilevers are each connected to a resistor on the substrate, defining a half Wheatstone bridge. Half of the cantilevers have a gold layer deposited on the top surface. This layer can be used for immobilization of biochemical entities containing sulphur. The gold coated cantilevers are used as the measurement cantilevers while the rest is used as references. Inserting one measurement and one reference cantilever in a Wheatstone bridge configuration, the reference cantilever subtracts noise from the surroundings. Integrated piezoresistive readout eliminates the use of bulky optical systems and it is possible to perform measurements in even nontransparent liquids.

The sensor principle is based on measuring a change in surface stress when molecules are attached to the surface of the stress-sensitive piezoresistor on the cantilever [4]. By functionalizing the cantilevers differently, it is possible to detect different bio-molecules in a complex sample. For example, it is possible to detect different samples of DNA and different types of antigens.

\section{THEORY}

A cantilever with piezoresistive readout gives a direct measure of the change in surface stress when molecules absorb on the cantilever surface. This is due to the fact that piezoresistors respond directly to stress changes. On the contrary, optical readout gives an indirect measure of the change in surface stress, since the surface stress is first transformed into a cantilever bending, which can then be measured.

A simple expression for surface stress sensitivity can be found assuming a cantilever consisting of only one material and an infinitely thin resistor placed on top of the cantilever.

When applying a surface stress $\sigma_{s}$ to the top surface of the cantilever, the stress distribution $\sigma(z)$ can be considered to increase linearly through the thickness of cantilever, see figure 2 . The neutral axis is placed at $z=0, \alpha$ is the relative distance from the neutral axis to the bottom of the cantilever, and $\sigma_{0}$ is the stress in the resistor. For this system it is required that both the net force $F$ and the net moment $N$ around the neutral axis are zero:

$$
\begin{aligned}
& F=w \cdot \sigma_{s}+w \cdot \int_{-\alpha h}^{(1-\alpha) h} \sigma(s) d z=0 \\
& N=(1-\alpha) h \cdot w \cdot \sigma_{s}+w \int_{-\alpha h}^{(1-\alpha) h} \sigma \cdot z d z=0
\end{aligned}
$$


where $w$ is the width of the cantilever and $h$ is the thickness of the cantilever. From these two equations, it is possible to extract an expression for the stress in the resistor $\sigma_{0}$ as a function of the surface stress $\sigma_{s}$. This expression is given in equation (3):

$$
\sigma_{0}=-\frac{4 \sigma_{s}}{h}
$$

The relative change in resistance for a piezoresistor can be expressed as $\frac{\Delta R}{R}=K \cdot \sigma / E$, where $K$ is the gauge factor of the piezoresitive materiale and $E$ is Youngs modulus of the cantilever materiale. Thus, the surface stress sensitivity can be expressed as the following:

$$
\frac{\Delta R}{R} / \sigma_{s}=-K \cdot \frac{4}{h \cdot E}
$$

From equation (4) it is seen that the surface stress sensitivity depends only on the gauge factor and the cantilever thickness and material.
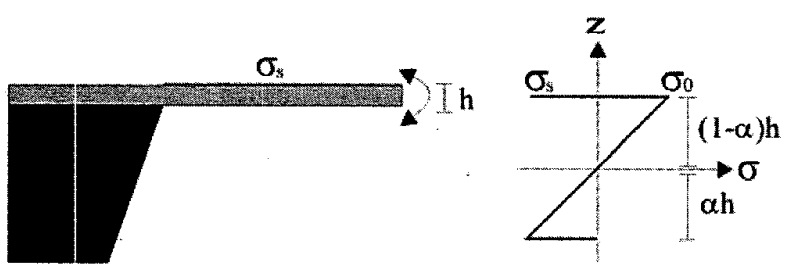

Figure 2: The schematic to the left shows a simple cantilever, with a thickness $h$, and an infinitely thin resistor on top. The cantilever is subjected to a surface stress $\sigma_{s}$. The graph to the right shows the stress distribution through the cantilever. A neutral axis is placed at $z=0$. The relative distance from the neutral axis to the bottom of the cantilever is given by $\alpha$.

Thus, in order to obtain a cantilever with very high surface stress sensitivity, the gauge factor should be high, the cantilever thin and the cantilever material soft. The cantilever length and the cantilever width have no influence on the sensitivity.

\section{FABRICATION}

The cantilever-based bio-chemical sensor is fabricated using conventional microfabrication techniques. Here we present cantilevers with poly-silicon resistors. Using single crystalline silicon resistors would give a better sensitivity and lower noise, and would therefore optimize the performance of the sensor. Nevertheless, the fabrication of single crystalline $\mathrm{Si}$ resistors involves the use of SOI.wafers, which makes the fabrication more complicated and more expensive.

First, a sandwich layer of silicon nitride and poly-silicon is deposited by low-pressure chemical deposition (LPCVD) on a 350 $\mu \mathrm{m}$ double-sided polished wafer (figure 3.a). Boron is implanted in the poly-silicon and the piezoresistors are defined by reactive ion etching $(R I E)$ (3.b). Next, a thick silicon nitride layer is deposited by $L P C V D$. The piezoresistor is now completely encapsulated (3.c) in silicon nitride is a very good diffusion barrier. The silicon nitride on the backside of the wafer is patterned by RIE in order to define liquid inlets and outlets and holes are subsequently etched in $\mathrm{KOH}$ leaving $100 \mu \mathrm{m}$ thick membranes (3.d). The cantilever, contact holes to the resistors and the channel are hereafter defined in the same mask step using a selective RIE (3.e). Gold, which enables immobilization of thiol-modified biochemical entities is deposited on the measurement cantilevers by lift-off technique (3.f). A $55 \mu \mathrm{m}$ deep channel is then etched using $\mathrm{KOH}$. The $\mathrm{KOH}$ etch releases the cantilevers and completes the definition of the liquid inlets and outlets. Next, aluminum is deposited on the wafer and patterned for the electrical connections (3.g). Epon SU-8 is used to define the 60 $\mu \mathrm{m}$ high upper part of the channel walls. Finally, a lid is placed on top of the channel using either laminating or gluing techniques leaving a perfectly sealed channel (3.h).

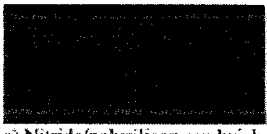

a) Nitridepolysilicon sandwiel

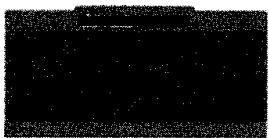

c) Thich L PCVD nitride

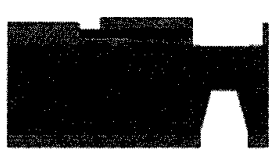

c) RIE of nitrid

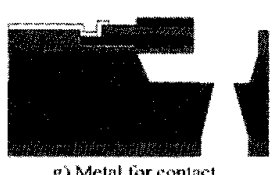

g) Metal lior tomatel

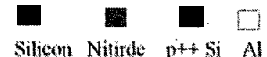

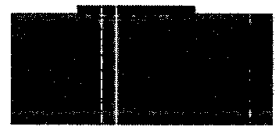

b) WEE of resistor

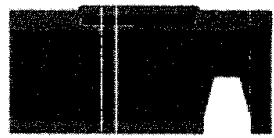

d) KOH the of contact

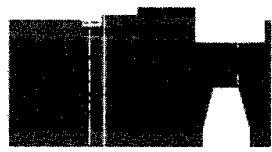

ค. Inum. metal

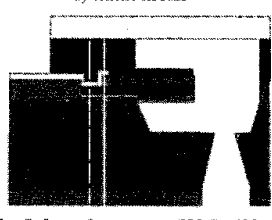

h) Spin and structure $S \mathrm{U}-\mathrm{z}+\mathrm{hid}$

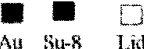

Figure 3: The process sequence for the cantilever-based biochemical sensor integrated in a micro-liquid handling system.

\section{CHARACTERIZATION}

Figure 4 shows a schematic side view of the sensor with two cantilevers in the channel. The piezoresistor is placed at the bottom of the cantilever and has a thickness of $150 \mathrm{~nm}$. The lower silicon nitride layer is $50 \mathrm{~nm}$ thick and the upper silicon nitride layer is 280 $\mathrm{nm}$ thick. The top chromium/gold layer is $40 \mathrm{~nm}$ thick, yielding a total cantilever thickness of $520 \mathrm{~nm}$. The cantilevers are rectangular and are $125 \mu \mathrm{m}$ long and $40 \mu \mathrm{m}$ wide. The channel is $400 \mu \mathrm{m}$ wide and $3 \mathrm{~mm}$ long, yielding a volume of $0.14 \mu \mathrm{l}$. Figure 5 shows SEM micrographs of the chip. The left micrograph shows the whole chip while the right micrograph shows the cantilevers in the channel From this micrograph it can be seen that the cantilevers are placed in the middle of the side wall.

The spring constant is calculated to $k=0.2 \mathrm{~N} / \mathrm{m}$ and the resonant frequency is $f=60 \mathrm{kHz}$. The gauge factor of the piezoresistor has been measured to 25 . Using a more complicated model than the one given above, the surface stress sensitivity is 
calculated to $\frac{\Delta R}{R}=4.6 \cdot 10^{-4}(\mathrm{~N} / \mathrm{m})^{-1}$. The more complicated model takes into consideration that the cantilever consists of a sandwich of different materials and that the resistor is placed in the cantilever and has finite dimensions.

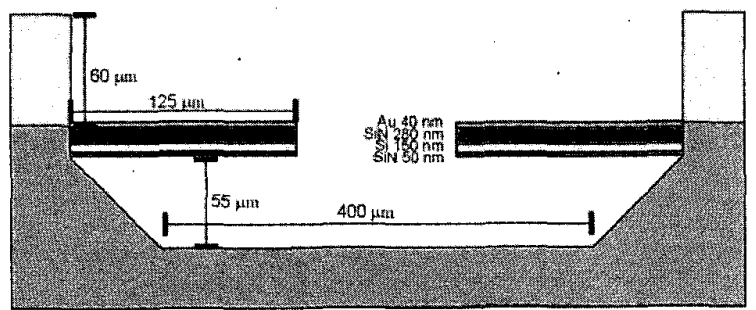

Figure 4: Schematic cross-section of two cantilevers in a channel. The top part of the channel wall is defined in Epon SU-8. The lower channel part is etched in silicon. The width of the channel is 400 $\mu \mathrm{m}$, and the length is $3 \mathrm{~mm}$. The cantilever width is $40 \mu \mathrm{m}$, the length is $125 \mu \mathrm{m}$ and the thickness is $520 \mathrm{~nm}$. The thickness of the individual layers is indicated on the right cantilever.

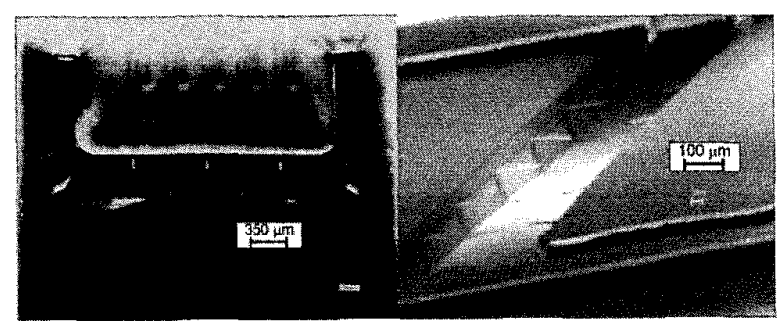

Figure 5: SEM micrographs of the sensor chip. The left image shows a top view of the whole channel with 10 cantilevers and 4 inlets/outlets. The right image shows a side-view of a part of the channel where it is possible to see that the thin cantilevers are placed in the middle of the sidewall.

The minimum detectable surface stress depends on the noise in the system. If external noise sources are neglected the main noise sources are Hooge noise ( $I / f)$ and Johnson noise (white noise) [5]. Figure 6, shows a plot of the noise power as a function of the frequency for different supply voltages. It can be seen that the Hooge noise is dependent on the Wheatstone bridge supply voltage, which is in correspondence with the theory [5]. The Johnson noise also depends on the supply voltage. This is in disagreement with the theory. A probable explanation is that the piezoresistor is selfheated and thereby changes the noise floor. Based on the noise measurements and the sensitivity of the sensor a minimum detectable surface stress change of $2.6 \mathrm{mN} / \mathrm{m}$ has been calculated.

In liquid, the measured noise level is lower than in air, which is probably due to damping of vibrations in the liquid. It does not matter with respect to noise, whether the measurement and the reference cantilever are placed next to each other or in opposite ends.

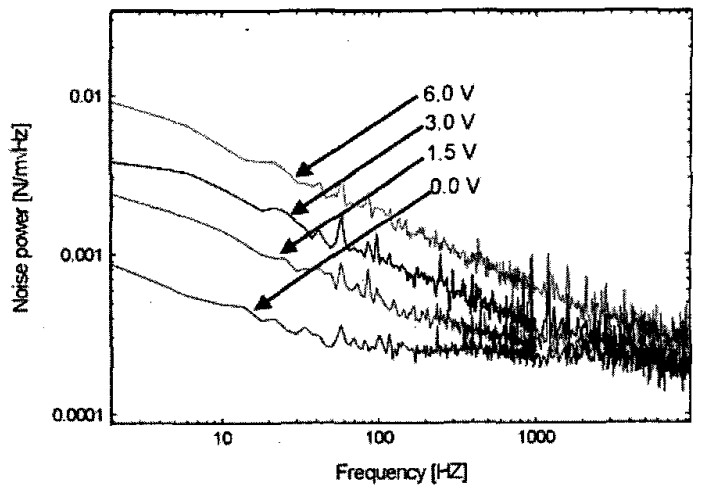

Figure 6: The noise power in a piezoresistive cantilever as a function of frequency. It is seen that both the Hooge noise and the Johnson noise is depended on the Wheatstone bridge supply voltage.

\section{APPLICATIONS}

The chip is mounted in and wire bonded to a chip house with four holes in the bottom for liquid connections. The chip house can then be placed in a setup where tubes are inserted from the backside, while the electrical connections are obtained from the frontside. The signal from the piezoresistive cantilevers, inserted in Wheatstone bridges, can then be read out simultaneously. The Wheatstone bridges are supplied by a voltage of $5 \mathrm{~V}$ and different liquids are pumped through the channel using syringe pumps and a valve. The pump rate is $20 \mu \mathrm{l} / \mathrm{min}$.

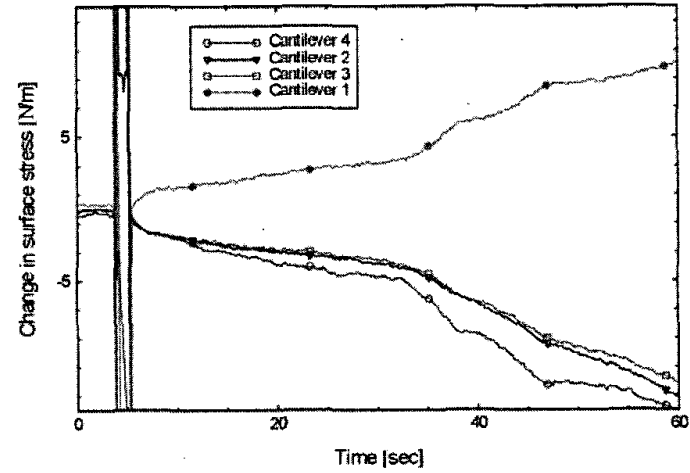

Figure 7: Surface stress change as a function of time for four goldcoated cantilevers subjected to a diluted aqua regia etch of the gold on the measurement cantilevers. The system is first purged with water. At $t=5$ seconds a bubble is observed, and then the aqua regia etch starts.

As mentioned previously, the Wheatstone bridge consists of two piezoresistive cantilevers, one for measurements and one for subtracting background noise. Since only the measurement cantilever has gold on the top surface, it is possible to study the effect of etching the gold in diluted aqua regia. In figure 7 , the surface stress change on four gold coated cantilevers is plotted as a function of time. First, DI-water is pumped through the system, and after 5 seconds a large signal due to an air bubble is observed. 
Immediately after the bubble, aqua regia is introduced into the system. The surface stress change momentarily on all cantilevers and all cantilevers respond identically. Cantilever 1 is placed in a Wheatstone bridge with a supply voltage of $-5 \mathrm{~V}$, whereas cantilever 2,3 and 4 are placed in Wheatstone bridges with a supply voltages of $+5 \mathrm{~V}$. Thus, the readout signal from cantilever 1 is opposite the signal from the rest of the cantilevers. The observed surface stress change corresponds to a removal of a top layer with the tensile stress of $35 \mathrm{MPa}$. This corresponds well with the normally observed stress in gold films.

The chip has also been used to measure the change in surface stress due to immobilization of thiol modified DNA-oligos. The thiol group is known to bind strongly to gold [6] whereas thiol will not bind to silicon nitride. After in-situ cleaning of the gold by a weak aqua regia solution, thiol modified DNA-oligos are introduced in the channel. Figure 8 shows the reaction of two gold coated cantilevers when the DNA-oligos are introduced. It is seen from the graph, that the change in surface stress is in the order of 3 $\mathrm{N} / \mathrm{m}$, and that the reaction stops after about 80 seconds, where we believe that a complete monolayer has been formed. After 40 seconds the two cantilevers stop to react equally. Maybe, the quality of the gold on the cantilevers is not the same due to the very rough cleaning/etching method used. This could easily result in different adsorption curves and thereby different rate constants. The surface stress change is found to follow a Langmuir type adsorption model [6], and the rate constants ( $k=0.03 / \mathrm{s}, k=0.05 / \mathrm{s})$ which describes the monolayer formation speed, are in good agreement with the literature [7].

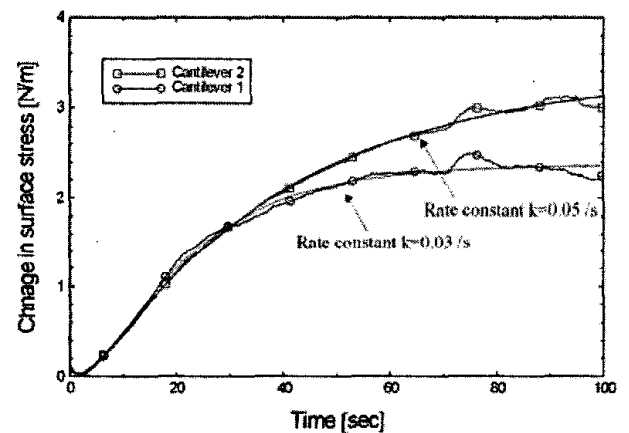

Figure 8: Surface stress changes as a function of time when a sample of thiol modified DNA-oligos are flowing in the channel. The DNA-oligo solution is introduced in the channel at $t=0$ seconds. The graphs both follow a Langmuir type adsorption model (smooth curves).

The chip has been designed with four liquid inlets/outlet in order to be able to flow two liquids in the channel at the same time. At reasonable flowrates $(<200 \mu \mathrm{l} / \mathrm{min})$ the flow in the channel can be considered laminar. In this regime it is possible to flow two different liquid samples side by side in the channel without significant mixing. The only mixing which occurs is due to diffusion. This principle can be used in several applications. For example, it is possible to flow a test sample in one part of the channel so only half the cantilevers are exposed to this sample, while a reference sample is flowing in the other part of the channel. Figure 9 shows how two liquids flow next to each other in the channel. The left liquid is dark. This could for example be used to monitor differences between two samples, if one cantilever from each flow were inserted in the same Wheatstone bridge.

\section{CONCLUSIONS}

Cantilevers with fully encapsulated piezoresistors have been integrated in micro-channels. The cantilevers can be operated safely in liquids with even high salt concentrations. The cantilever-based sensor offers the opportunity of in-situ monitoring of etching and immobilisation processes and yields a direct measure of the stress change in the cantilevers surface layer. Such a system offers an attractive direct electrical read-out from even non-transparent liquids, avoiding bulky optical read-out. Thus, the detection principle described here makes it possible to develop compact biosensors with a simple read-out scheme.

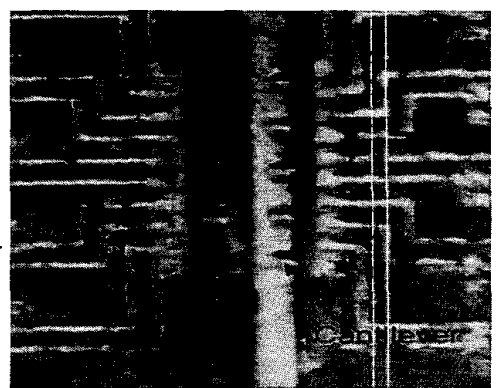

Figure 9: Optical microscope image of the channel. The channel is seen from above. Two laminated liquids are flowing side by side. One is dark and one is bright.

\section{REFERENCES}

1. J. Fritz, M.K. Baller, H.P. Lang, H.Rothuizen, P. Vettiger, E.Meyer, H. Güntherodt, Ch. Gerber, J.K.Gimzewski, "Translating biomolecular recognition into nanomechanics" Science, Vol. 288 2000 April 14, pp. 316-318

2. S.J. Shea, T.A. Brunt, A.R. Ramadan, T. Raymetn, and M.E. Welland, "Atomic force microscopy stress sensors for studies in liquids", J. Vac. Sci. Technol. B 14 (2) 1996 pp. 1383-1385

3. R. Raiteri, W. Knoll, P. Skádal G. Nelles, and H. Butt. "A novel transducer for direct sensing of bioaffinity interactions based on microfabricated cantilevers", J. Phys, Chem, 1995, 99, pp. 15728 15732

4. A. Boisen, J. Thaysen, H. Jensenius, and O. Hansen, "Environmental sensors based on micromachined cantilevers with integrated read-out", Ultramicroscopy 82 (2000) pp. 11-16

5. P.A. Rasmussen, J. Thaysen, S. Bouwstra and A. Boisen "Modular design of AFM probe with sputtered silicon tip", submitted to Sensors and Actuators in August 2000

6. R. Berger, E. Delamarche, H.P. Lang, C. Gerber, J. Gimzewski E. Meyer, H. Güntherodt, "Surface stress in self-assembly of alkanethiols on gold probed by a force microscopy technique", Appl. Phys. A 66, 1998, pp. 55-59

7. M. Yang, H.C.M. Yau and H.L. Chan, "Adsorption kinetics and ligand-binding properties of thiol-modified double stranded DNA on gold surface, Langmuir, 14, 1998, pp. 6121-6129 